\title{
Ácidos grasos de lípidos intramusculares y grasa de cobertura en búfalos suplementados con aceite de pescado
}

\author{
Cedrés, J.F.'; Rébak, G.I. ${ }^{1}$ Patiño, E.M. ; Sánchez Negrette, M. ${ }^{1}$; Tuñón, G. ${ }^{2}$
}

${ }^{1}$ Facultad de Ciencias Veterinarias, Universidad Nacional del Nordeste, Sargento Cabral 2139, Corrientes (3400), Argentina. ${ }^{2}$ New Zealand Farming Systems Uruguay.

E-mail: cedres@vet.unne.edu.ar

\begin{abstract}
Resumen
Cedrés, J.F.; Rébak, G.I.; Patiño, E.M.; Sánchez Negrette, M.; Tuñón, G.: Ácidos grasos de lípidos intramusculares y grasa de cobertura en búfalos suplementados con aceite de pescado. Rev. vet. 24: 2, 124-128, 2013. El objetivo fue investigar la influencia de la suplementación dietaria de búfalos con aceite de pescado sobre el ácido linoleico conjugado (ALC) y los ácidos grasos $\Omega 6$ y $\Omega 3$ en lípidos intramusculares (IM) y grasa de cobertura (GC). Se emplearon veinte animales divididos en dos grupos alimentados sobre Brachiaria brizantha y suplementados con $3 \mathrm{~kg}$ de afrecho de arroz, $500 \mathrm{~g}$ de maíz y $500 \mathrm{~g}$ de pellet de girasol, durante 60 días. El grupo I se mantuvo con esta dieta, el II recibió adicionalmente $100 \mathrm{ml} / \mathrm{día}$ de aceite de pescado por animal. Los resultados (expresados en $\mathrm{mg} / \mathrm{g}$ grasa) indicaron una disminución de ácido palmítico en IM y GC del grupo II $(189,05)$ vs grupo I $(206,97)$ y en II $(259,72)$ vs I $(280,84)$ respectivamente. El ALC se incrementó levemente en IM y GC del grupo II $(17,09)$ vs I $(11,56)$ y en el II $(16,80)$ vs I $(11,75)$ respectivamente. El ácido linoleico $(18: 2 \mathrm{n} 6)$ en IM del grupo II $(23,45)$ disminuyó en relación con el I $(38,19)$ y en GC del grupo II $(13,21)$ se incrementó levemente con relación al I $(12,71)$. El ácido alfa linolénico (18:3 n3) en IM de grupos I y II fue similar $(8,37$ y 8,69$)$, en cambio el contenido de dicho ácido en GC fue menor en el grupo I $(4,70)$ comparado con el grupo II $(6,05)$. La relación $\Omega 6 / \Omega 3$ en IM y GC fue más estrecha en el grupo II $(2,69: 1)$ y $(2,18: 1)$ con relación al grupo I $(4,55: 1)$ y I $(2,70: 1)$ respectivamente. La suplementación con aceite de pescado produjo disminución del ácido linoleico, mejorando la relación entre $\Omega 6 / \Omega 3$. Además causó una leve disminución de los valores de ácido palmítico y un pequeño aumento de ALC.
\end{abstract}

Palabras clave: búfalo, ácidos grasos, lípidos intramusculares, grasa de cobertura, suplementación con aceite de pescado.

\begin{abstract}
Cedres, J.F.; Rebak, G.I.; Patiño, E.M.; Sánchez Negrette, M.; Tuñon, G.: Fatty acids of intramuscular lipids and subcutaneous fat in buffaloes supplemented with fish oil. Rev. vet. 24: 2, 124-128, 2013. The purpose of this study was to investigate the influence of fish oil as dietary supplement on CLA and $\Omega 6$ and $\Omega 3$ fatty acids on both intramuscular lipids (IM) and layer fat (LF) in buffaloes. Twenty animals were randomly divided into two groups, fed on Brachiaria brizantha, $3 \mathrm{~kg}$ rice bran, $500 \mathrm{~g}$ corn and $500 \mathrm{~g}$ sunflower pellets for 60 days. Group I only received this diet while in group II each animal received the diet plus 100 $\mathrm{ml}$ fish oil every day. Results (in $\mathrm{mg} / \mathrm{g}$ fat) indicated a decrease of palmitic acid in group II (189.05) in relation to group I (206.97) in IM and in the group II (259.72) in relation to group I (280.84) in LF. In IM, the CLA content of group II (17.09) showed an increase compared to group I (11.56). In LF the CLA content in group II (16.80) showed an increase in relation to group I (11.75). In IM the linoleic acid (18:2 n6) content of group II (23.45) decreased in relation to group I (38.19) and LF in the group II (13.21) increased slightly in relation to group I (12.71). In IM alfa linolenic acid (18:3 n3) content of groups I and II was very similar (8.37 and 8.69 respectively) and in LF was of 4.70 and 6.05 respectively. In IM of group II $\Omega 6 / \Omega 3$ ratio was narrower (2.69:1) than in group I (4.55:1). Fish oil supplementation caused decrease of linoleic acid, improving the $\Omega 6 / \Omega 3$ relationship. Furthermore, it caused a slight decrease of palmitic acid and slight increase of CLA.
\end{abstract}

Key words: buffalo, fatty acids, intramuscular and layer fat, fish oil supplementation. 


\section{INTRODUCCIÓN}

El término genérico de alimento funcional se utiliza para identificar alimentos y/o componentes de los mismos que posean propiedades adicionales sobre la salud de los consumidores, superando el beneficio clásico de un aporte de nutrientes ${ }^{10}$.

Si bien la grasa de los productos bovinos tanto en leche como en carne en muchos casos es considerada perjudicial para la salud por su alto contenido en ácidos grasos saturados, en los últimos años se ha encontrado que un componente de las mismas, el ácido linoleico conjugado (ALC), posee propiedades anticancerígenas ${ }^{14} \mathrm{y}$ actividad lipolítica preventiva de arteriosclerosis y diabetes, por lo cual todo alimento con alto contenido de ALC es considerado como "funcional" 10, 12 .

Los productos alimenticios provenientes de rumiantes constituyen una de las mayores fuentes dietarias de ALC para seres humanos y asumen importancia en la producción de alimentos funcionales ${ }^{1}$. El ALC se forma en el rumen como intermediario derivado de la biohidrogenación del ácido linoleico por la enzima ácido linoleico isomerasa, producida por bacterias anaeróbicas del genero Butyrivibrio, que transforman al referido ácido graso en un isómero cis-9 y trans-11 17 .

Las fuentes principales de ALC en la dieta humana son la leche y la carne de los rumiantes, las cuales contienen principalmente cis-9, trans-11 C18:2 (ácido ruménico) y trans-9, cis-11 C18:2; el primero contribuye al $60 \%$ del ALC total en el músculo ${ }^{11}$. Investigaciones que determinaron ALC en carne de ganado bubalino en países como Italia ${ }^{20}$, Brasil ${ }^{15,16,19}$, Venezuela ${ }^{6} \mathrm{y}$ Argentina, obtuvieron valores disímiles debido a las diferentes dietas empleadas.

Se ha demostrado que los ácidos grasos eicopentanoico y docosahexanoico $\Omega 3$ tienen propiedades hipocolesterolémicas, antitrombóticas y antiinflamatorias ${ }^{24}$. Varios autores consideran que para la salud humana, es conveniente utilizar la relación $\Omega 6 / \Omega 3$ entre 5 y 2 o similares ${ }^{5}$. Las dietas con esta relación deberían considerarse "alimentos funcionales".

La población de búfalos en Argentina se estima en 100.000 cabezas, concentradas mayoritariamente en el

Tabla 1. Composición del aceite de pescado utilizado.

\begin{tabular}{lc}
\hline contenido de ácidos grasos & $\%$ \\
\hline ácido alfa linoleico 18:3 n-3 & 1,10 \\
ácido estearico 18:4 n-3 & 2,58 \\
ácido eicosatrienoico 20:3 n-3 y n-6 & 0,16 \\
ácido araquidónico 20:4 n-3 & 0,62 \\
ácido eicosapentaenoico EPA 20:5 n-3 & 7,18 \\
ácido eneicosapentaenoico 21:5 n-3 & 0,23 \\
ácido docosapentanoico DPA 22:5 n-3 & 0,70 \\
ácido docosahexaenoicoDHA 22:6 n-3 & 16,47 \\
ácidos grasos $\Omega$ 3 & 29,04 \\
ácidos grasos $\Omega$ 6 & 3,52 \\
ácidos grasos poliinsaturados & 32,56 \\
\hline
\end{tabular}

subtrópico húmedo de la zona nordeste, en las provincias de Corrientes, Chaco, Misiones, Formosa y norte de Santa Fe, siendo Corrientes la que posee mayor cantidad, calculadas en 45.000 cabezas. También se crían búfalos en otras provincias como Buenos Aires, Entre Ríos, Tucumán, Mendoza y San Luis. La producción de carne es el principal objetivo de la cría de esta especie.

El propósito del presente trabajo fue investigar si la suplementación con aceite de pescado es capaz de incrementar el ALC y modificar la relación $\Omega 6 / \Omega 3$ en lípidos intramusculares y grasa de cobertura de búfalos mantenidos sobre pradera de Brachiaria brizantha.

\section{MATERIAL Y MÉTODOS}

Los animales utilizados pertenecían a un establecimiento ubicado en el departamento General Paz, Provincia de Corrientes, Argentina. Se trabajó con 20 bubillos castrados de 11 meses de edad de raza Mediterránea, identificados con caravanas alfanuméricas, distribuidos en dos grupos integrados por diez ejemplares cada uno. Durante los 60 días que duró la experiencia, todos los animales fueron alimentados con pasto $\mathrm{Bra}$ chiaria brizantha y una ración diaria por animal de 3 $\mathrm{kg}$ de afrecho de arroz, $500 \mathrm{~g}$ de maíz y $500 \mathrm{~g}$ de pellets de girasol (el contenido de extracto etéreo fue de 14,4\% de la materia seca). Los animales del grupo I (control) se alimentaron exclusivamente con pasto y ración; los del grupo II recibieron además diariamente $100 \mathrm{ml}$ de aceite de pescado mezclado con la ración.

La composición de los ácidos grasos del aceite de pescado se consigna en la Tabla 1; el $85 \%$ del aceite provenía de merluzas (Merluccis hubbsi) y el 15\% restante de anchoas (Anchoa marinii).

Cabe aclarar que el grupo II opuso resistencia a comer la totalidad de la ración durante los primeros días, debido a que el aceite de pescado estaba desodorizado pero no saborizado. De los pesajes del sobrante de los comederos surgió que durante los primeros 30 días de ensayo los animales solo consumieron el $50 \%$ de la ración. El peso medio de los bubillos $(\mathrm{n}=20)$ al inicio del ensayo fue de $278 \pm 22 \mathrm{~kg}$ (rango $245-320 \mathrm{~kg}$ ) y al final de la experiencia fue de $323 \pm 32 \mathrm{~kg}$ (rango $245-384 \mathrm{~kg}$ ).

Las muestras $(\mathrm{n}=20)$ de los lípidos intramusculares del músculo longissimus dorsi y de la grasa de cobertura adosada al bife angosto entre la $10^{\circ}$ y $11^{\circ}$ costillas, fueron obtenidas al día 60 de la experiencia, entre los meses de febrero y marzo de 2011. Las muestras fueron acondicionadas en recipientes descartables, congeladas a $-20^{\circ} \mathrm{C}$ y mantenidas en cajas de poliuretano hasta su llegada al laboratorio.

Cada muestra fue procesada por duplicado para la obtención del perfil lipídico. Para extraer los lípidos totales se utilizó una mezcla de cloroformo y metanol de acuerdo con la técnica de Bligh and Dyer ${ }^{3}$ manteniendo atmósfera de nitrógeno. La conversión de los ácidos grasos en metilésteres se llevó a cabo con $\mathrm{NaOH}$ y $\mathrm{BF}_{3}$ metanólico al $14 \%$ a ebullición durante 8 minutos. Los metilésteres se extrajeron con hexano y se analizaron 
Tabla 2. Perfil de ácidos grasos (mg/g de grasa) en lípidos intramusculares según grupos.

\begin{tabular}{|c|c|c|c|c|c|c|c|}
\hline \multirow{2}{*}{ ácidos grasos } & \multicolumn{3}{|c|}{ grupo I (pastura) } & \multicolumn{3}{|c|}{ grupo II (pescado) } & \multirow{3}{*}{$\begin{array}{c}\mathrm{CV}(\%) \\
27,56\end{array}$} \\
\hline & \multirow{2}{*}{$\begin{array}{c}\mathrm{n} \\
10\end{array}$} & \multirow{2}{*}{$\begin{array}{c}\text { media } \\
15,48^{\text {a }}\end{array}$} & \multirow{2}{*}{$\begin{array}{c}\mathrm{EE} \\
1,54\end{array}$} & \multirow{2}{*}{$\begin{array}{c}\mathrm{n} \\
10\end{array}$} & \multirow{2}{*}{$\begin{array}{c}\text { media } \\
16,98^{\text {a }}\end{array}$} & \multirow{2}{*}{$\begin{array}{c}\mathrm{EE} \\
1,54\end{array}$} & \\
\hline $\begin{array}{l}\mathrm{S} \quad(14: 0) \text { mirístico } \\
\end{array}$ & & & & & & & \\
\hline S $\quad(15: 0)$ pentadecanoico & 4 & $1,72^{\text {a }}$ & 0,38 & 6 & $2,23^{\mathrm{a}}$ & 0,31 & 16,98 \\
\hline S (16:0) palmítico & 10 & 206,97 a & 11,20 & 10 & $189,05^{\text {a }}$ & 11,20 & 17,71 \\
\hline S $\quad(17: 0)$ heptadecanoico & 10 & $13,82^{\mathrm{a}}$ & 1,49 & 10 & $14,34^{\mathrm{a}}$ & 1,49 & 27,73 \\
\hline S (18:0) esteárico & 10 & $299,42^{\text {a }}$ & 32,83 & 10 & $293,83^{a}$ & 32,83 & 27,24 \\
\hline S $\quad(20: 0)$ araquidónico & 5 & $2,28^{a}$ & 0,50 & 7 & $3,32^{\text {a }}$ & 0,42 & 23,47 \\
\hline M (16:1) palmitoleico & 10 & $17,67^{\text {a }}$ & 1,55 & 10 & $17,46^{\mathrm{a}}$ & 1,55 & 23,74 \\
\hline M $(17: 1)$ heptadecenoico & 10 & $7,59^{\text {a }}$ & 0,80 & 10 & $6,29^{\text {a }}$ & 0,80 & 32,38 \\
\hline $\mathrm{M} \quad(18: 1) \mathrm{n} 9 \mathrm{t}$ vacénico & 9 & $9,14^{\text {a }}$ & 0,25 & 10 & $14,13^{\text {a }}$ & 0,25 & 49,27 \\
\hline M $\quad(18: 1)$ n9 c oleico & 10 & $366,22^{\text {a }}$ & 23,55 & 10 & $308,44^{a}$ & 23,55 & 21,57 \\
\hline $\mathrm{P} \quad(18: 2) \mathrm{n} 6 \mathrm{c}$ linoleico & 10 & $38,19^{\text {a }}$ & 4,72 & 10 & $23,45^{b}$ & 4,72 & 46,64 \\
\hline $\mathrm{P} \quad(18: 3) \mathrm{n} 3 \alpha$ linolénico & 10 & $8,37^{\text {a }}$ & 0,94 & 10 & $8,69^{\text {a }}$ & 0,94 & 32,64 \\
\hline $\mathrm{P} \quad(18: 2) 9 \mathrm{c}, 11 \mathrm{t}$ ALC & 9 & $11,56^{\mathrm{a}}$ & 2,23 & 10 & $17,09^{\text {a }}$ & 2,36 & 46,30 \\
\hline
\end{tabular}

S: saturado, M: monoinsaturado, P: poliinsaturado, EE: error estándar, CV: coeficiente de variación. Letras diferentes indican diferencias significativas entre medias $(\mathrm{p}<0,05)$.

Tabla 3. Perfil de ácidos grasos (mg/g de grasa) en grasa de cobertura según grupos.

\begin{tabular}{llccccccc}
\hline \multirow{2}{*}{ ácidos grasos } & \multicolumn{3}{c}{ grupo I (pastura) } & \multicolumn{3}{c}{ grupo II (pescado) } & \multirow{2}{*}{ CV(\%) } \\
\cline { 2 - 7 } S & $(14: 0)$ mirístico & $\mathrm{n}$ & media & $\mathrm{EE}$ & $\mathrm{n}$ & media & EE & \\
$\mathrm{S}$ & $(10$ & 26,49 & 2,19 & 10 & 28,65 & 2,19 & 23,44 \\
$\mathrm{~S}$ & $(16: 0)$ pentadecanoico & 10 & 5,12 & 0,42 & 10 & 5,38 & 0,42 & 22,96 \\
$\mathrm{~S}$ & $(17: 0)$ heptadético & 10 & 280,84 & 19,10 & 10 & 259,72 & 19,10 & 20,57 \\
$\mathrm{~S}$ & $(18: 0)$ esteárico & 10 & 20,53 & 1,54 & 10 & 18,33 & 1,54 & 22,22 \\
$\mathrm{~S}$ & $(20: 0)$ araquidónico & 10 & 251,92 & 51,0 & 10 & 279,90 & 51,10 & 29,13 \\
M & $(16: 1)$ palmitoleico & 10 & 4,81 & 0,48 & 10 & 5,25 & 0,54 & 47,88 \\
M & $(18: 1)$ n9 t vacénico & 10 & 17,90 & 1,97 & 10 & 16,33 & 1,97 & 32,21 \\
M & $(18: 1)$ n9 c oleico & 10 & 31,17 & 2,86 & 10 & 34,71 & 2,86 & 24,22 \\
P & $(18: 2)$ n6 c linoleico & 10 & 332,06 & 28,25 & 10 & 315,67 & 28,85 & 25,24 \\
P & $(18: 3)$ n3 $\alpha$ linolénico & 10 & 12,71 & 1,23 & 10 & 13,21 & 1,23 & 26,42 \\
P & $(18: 2)$ 9c,11t ALC & 10 & 4,70 & 0,49 & 10 & 6,05 & 0,49 & 26,96 \\
\hline
\end{tabular}

S: saturado, M: monoinsaturado, P: poliinsaturado, EE: error estándar, CV: coeficiente de variación. No hubo diferencias significativas entre medias de ambos grupos $(\mathrm{p}<0,05)$.

con un cromatógrafo de fase gaseosa. Se utilizaron estándares de metilésteres de ácidos grasos de 99\% de pureza (Lipid Standard 189-19 Sigma-Aldrich). La composición de ácidos grasos se obtuvo en un cromatógrafo de fase gaseosa de la firma Agilent equipado con una columna capilar de $60 \mathrm{~m}$ de largo y $0,25 \mathrm{~mm}$ de diámetro interno (Supelco 2340) y un detector de ionización de llama. El método de cromatografía gaseosa utilizado (GC-FID) se adecuó a la norma ISO $15304^{8}$.

Se efectuaron estadísticas descriptivas para estimar los resultados de cada uno de los tratamientos (media aritmética, error estándar y coeficiente de variación). El análisis de la variancia se realizó bajo un diseno completamente aleatorizado, con un modelo lineal aditivo $\left(X_{i j}=\mu+\tau_{j}+e_{i j}\right)$, comprobando previamente los supuestos básicos de homogeneidad y normalidad. Los cálculos fueron realizados con el auxilio del software Infostat (2009), de propiedad de la Facultad de Ciencias Veterinarias de la Universidad Nacional del Nordeste.

\section{RESULTADOS Y DISCUSIÓN}

La composición de los ácidos grasos tanto de los lípidos intramusculares (IM) como de la grasa de cobertura (GC) se presenta en las Tablas 2 y 3 respectivamente. Expresando los valores en $\mathrm{mg} / \mathrm{g}$ grasa, el ácido graso palmítico en IM del grupo II $(189,05)$ resultó numéricamente inferior al del grupo I $(206,97)$; lo mismo sucedió en GC con el grupo II $(259,72)$ con respecto al grupo I $(280,84)$. Estos resultados si bien no fueron estadísticamente significativos, son dignos de consideración debido a que dicho ácido graso, conjuntamente con otros ácidos saturados como láurico y mirístico, poseen efecto hipercolesterolémico y su aumento constituye un factor de riesgo para el desarrollo de lesiones ateromatosas ${ }^{5,24}$.

En cuanto al contenido de ALC (18:2) 9c 11t tanto en IM como en GC del grupo II (17,09 y 16,80 respectivamente), resultaron superiores con respecto a los valo- 
Tabla 4. Composición (\%) de ácidos grasos en lípidos intramusculares (IM) y grasa de cobertura (GC).

\begin{tabular}{lcccc}
\hline \multirow{2}{*}{ ácidos grasos } & \multicolumn{2}{c}{ grupo I (pasto) } & \multicolumn{2}{c}{ grupo II (pescado) } \\
\cline { 2 - 5 } & IM & GC & IM & GC \\
\hline saturados & 55,92 & 58,97 & 58,08 & 59,72 \\
insaturados & 44,08 & 41,03 & 41,92 & 40,28 \\
monoinsaturados & 39,29 & 38,11 & 36,43 & 36,67 \\
poliinsturados & 4,79 & 2,92 & 5,49 & 3,61 \\
\hline
\end{tabular}

Tabla 5. Relación $\Omega 6 / \Omega 3$ en lípidos intramusculares (IM) y grasa de cobertura (GC).

\begin{tabular}{|c|c|c|c|c|c|c|}
\hline \multirow{2}{*}{ grupo } & \multicolumn{2}{|c|}{$\begin{array}{c}\Omega 6 \\
\text { (mg/g grasa) }\end{array}$} & \multicolumn{2}{|c|}{$\begin{array}{c}\Omega 3 \\
\text { (mg/g grasa) } \\
\end{array}$} & \multicolumn{2}{|c|}{$\begin{array}{c}\text { relación } \\
\Omega 6 / \Omega 3\end{array}$} \\
\hline & $\mathrm{IM}$ & $\mathrm{GC}$ & IM & $\mathrm{GC}$ & IM & GC \\
\hline I (pasto) & 38,19 & 12,71 & 8,37 & 4,70 & $4,55: 1$ & $2,70: 1$ \\
\hline II (pescado) & 23,45 & 13,21 & 8,69 & 6,05 & 2,69:1 & $2,18: 1$ \\
\hline
\end{tabular}

res del grupo I (11,56 y 11,75 respectivamente), aunque estos incrementos no fueron estadísticamente significativos. Resulta muy importante poder incrementar la concentración del ALC en un producto de consumo humano debido a sus potenciales efectos benéficos, tales como disminución de la formación de lesiones ateromatosas y propiedades antiinflamatoria y anticancerígena, comprobadas experimentalmente en modelos biológicos 7, 9, 13,21.

En IM el contenido de ácido linoleico (18:2 n6) del grupo II $(23,45)$ disminuyó significativamente comparado con el del grupo I $(38,19)$. Tal disminución es importante porque permite estrechar la relación $\Omega 6 /$ $\Omega 3$. Para la salud humana, el consumo de productos con altas concentraciones de $\Omega 6$ se asocia con trombosis, ateromatosis, desordenes alérgicos e inflamatorios, mientras que el consumo de alimentos ricos en $\Omega 3$ se relaciona con disminución de la formación de trombos y reducción de la ateromatosis, a los que se suman propiedades antiinflamatorias, hipocolesterolémicas y anticancerígenas 2, 4, 18, 21, 22, 24 .

La suplementación con aceite de pescado, si bien no aumentó la concentración de $\Omega 3$ como era esperado, produjo una disminución de las concentraciones de $\Omega 6$. En GC el valor de $\Omega 6$ obtenido en el grupo II $(13,21)$ se incrementó ligeramente en comparación con el grupo I $(12,71)$ sin que estos resultados fueran estadísticamente significativos. Respecto al ácido alfa linolenico (18:3 n3) en IM los valores obtenidos para los grupos I y II fueron similares, de 8,37 y 8,69 respectivamente y en GC fueron diferentes entre el grupo I $(4,70)$ y II $(6,05)$, no registrándose diferencias significativas.

Los porcentajes de ácidos grasos saturados, insaturados, monoinsaturados y poliinsaturados de cada grupo en IM y GC son exhibidos en la Tabla 4. La relación de los ácidos grasos $\Omega 6 / \Omega 3$ se presenta en la Tabla 5 . En IM la relación de los ácidos grasos $\Omega 6 / \Omega 3$ del grupo II fue mas estrecha $(2,69: 1)$ que la del grupo I $(4,55: 1)$. En GC esta relación fue también mas estrecha en el grupo II $(2,18: 1)$ que en la del grupo I (2,70:1) aunque sin diferencia significativa. Pese a ello, se considera que el aporte dietario del aceite de pescado fue importante porque estrechó la relación $\Omega 6 / \Omega 3^{23}$.

Los valores de ALC en GC e IM obtenidos en la presente experiencia, tanto en los bubillos alimentados a pastura como suplementados con aceite de pescado, fueron superiores a los registrados en Brasil ${ }^{16}$ con bubillos Murrah castrados mantenidos en confinamiento con tres tipos de dietas: sin aceite adicional, grano de soja integral y aceite de soja $(2,20-2,10-4,60 \mathrm{mg} / \mathrm{g}$ de lípidos intramusculares) y $(1,60-2,50-3,40 \mathrm{mg} / \mathrm{g}$ de grasa de cobertura) respectivamente para cada tipo de dieta. Nuestros valores de ALC demuestran la importancia de la pastura como base para la suplementación con aceite de pescado. Por su parte, la relación $\Omega 6 /$ $\Omega 3$ fue más estrecha $(2,18: 1)$ en el grupo II, que recibió aceite de pescado.

En conclusión, la suplementación de búfalos con aceite de pescado provocó cambios en los lípidos intramusculares y en la grasa de cobertura, consistentes en disminución significativa de ácido linoleico, mejoría de la relación entre $\Omega 6 / \Omega 3$ y leve reducción de los valores de ácido palmítico, aunque los aumentos de ácido linoleico conjugado no fueron significativos.

Agradecimientos. Al establecimiento "Florencia" de la localidad de General Paz, Corrientes, Argentina por aportar instalaciones y animales para el presente ensayo. A la empresa Omega Sur (Batan, Argentina), por proveer el aceite de pescado.

\section{REFERENCIAS}

1. Barcelli UO, Glass-Greenwalt P, Pollak VE. 1985. Enhancing effect of dietary supplementation with omega-3 fatty acids on plasma fibrinolysis in normal subjects. Tromb Res 39: 307-312.

2. Bauman DE, Baumgard LH, Corl BA, Griinari JM. 1999. Biosynthesis of conjugated linoleic acid in ruminants. Proc Am Soc Anim Sci 4: 1-11.

3. Bligh EG, Dyer WJ. 1959. A rapid method for total lipid extraction and purification. Can J Biochem Physiol 37: 911-917.

4. Brown AL, Roberts DC. 1991. Fish and fish oil intake: effect on hematological variables related to cardiovascular disease. Tromb Res 64: 169-178.

5. Gagliostro GA. 2004. Manejo nutricional para la producción de leches de vaca y de cabra con alto impacto sobre la salud humana. Publ. INTA (Balcarce, Argentina), $84 \mathrm{p}$.

6. Giuffrida M, Arenas L, Beriain MJ, Huerta NO, Smith GC. 2005. Occurrence of conjugated linoleic acid in longissimus muscle of water buffalo (Bubalus bubalis) and zebu-type cattle raised under savannah conditions. Meat Sci 69: 93-100.

7. Ip C, Lisk DJ, Scimeca JA. 1994. Potential of food modification in cancer prevention. Cancer Res 54: 1957-1959. 
8. ISO (Organización Internacional para la Estandarización). 2002. Norma 15304. Animal and vegetable fats and oils. Determination of the content of trans fatty acid isomers of oils. http://www.iso.org/iso/catalogue_detail. htm?csnumber $=35454$.

9. Kritchevsky D, Tepper SA, Wright S, Tso P, Czarnecki SK. 2000. Influence of conjugated linoleic acid (CLA) on establishment and progression of atherosclerosis in rabbits. J Am Coll Nutr 19: 472-477.

10. Lira GM, Mancini J, Torres RP, Oliveira AC, Vasconcelos AM, Omena CM, Almeida, MC. 2005. Composição centesimal, valor calórico, teor de colesterol e perfil de ácidos graxos da carne de búfalos (Bubalus bubalis) da cidade de Sao Luiz do Quintunde (Brasil). Rev Ins Adolfo Lutz 64: 31-38

11. McGuire MA, McGuire MK. 2000. Conjugated linoleic acid (CLA): a ruminant fatty acid with benefical effects on human health. Proc Am Soc Anim Sci 99: 1-8.

12. Milner JA. 1999. Functional foods and health promotion. J Nutr 129: 1395-1397.

13. Monson FD. 2004. Suplementación con lípidos en bovinos de carne: metabolismo, efectos sobre la calidad de la canal, de la carne y sobre la salud humana. Tesis Doctoral, Instituto Mediterráneo de Zaragoza (España), $110 \mathrm{p}$.

14. Nagao K, Yanagita T. 2005. Conjugated fatty acids in food and their health benefits. J Biosci Bioeng 100: 152157.

15. National Research Council (NRC). 1996. Carcinogenesis and anticarcinogenesis in the human diet, National Academy Press (Washington), $242 \mathrm{p}$.
16. Oliveira RL, Ladeira MM, Barbosa MA, Assunção DM, Matsushita M, Santos GT, Oilveira RL. 2008. Ácido linoléico conjugado e perfil de ácidos graxos no músculo e na capa de gordura de novilhos bubalinos alimentados com diferentes fontes de lipídios. Arq Bras Med Vet Zootec 60: 169-178.

17. Parodi PW. 1977. Conjugated octadecaienoic acids of milk fat. J Dairy Sci 60: 1550-1553.

18. Reddy B, Suggie S. 1988. Effect of differents levels of omega-3 and omega- 6 fatty acids on azoximethane induced colon carcinogenesis in F344 rats. Cancer Res 48 : 6642-6647.

19. Rodrigues VC, Bressan MC, Cardoso MG, Freitas RT. 2004. Ácidos graxos na carne de búfalos e bovinos castrados e inteiros. Rev Bras Zootec 23: 434-443.

20. Romano R, Borriello I, Chianese L, Sachi R. 2007. Efect of dietary vitamin E content on the CLA, cholesterol and triglycerides composition of Italian Mediterranean buffalo. Ital J Anim Sci 6: 1202-1206.

21. Sailas B, Spener F. 2009. Conjugated linoleic acids as functional food: an insight into their health benefits. $\mathrm{Nu}$ trition \& Metabolism 6: 36.

22. Simopoulos AP. 2002. The importance of the ratio of omega-6/omega-3 essential fatty acids. Biomed \& Pharmacother 56: 365-379.

23. Sperling R, Benincaso A, Knoell C, Larkin J, Austen K, Robinson D. 1993. Dietary omega-3 polyunsaturated fatty acids inhibit phosphoinositide formation and chemotaxis in neutrophils. $J$ Clin Invest 91: 651-660.

24. Williams CM. 2000. Dietary fatty acid and human health. Ann Zootech 49: 165-180. 